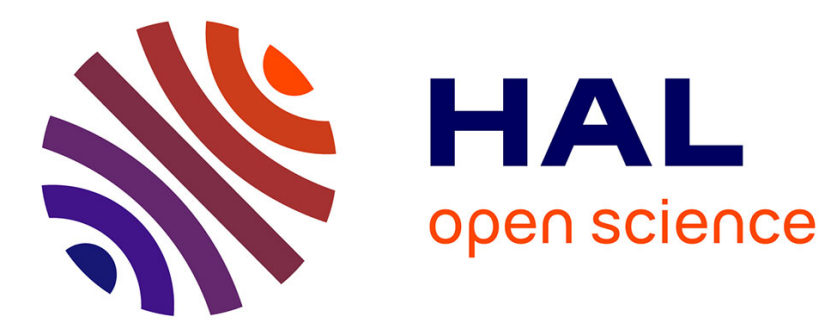

\title{
Comparison of Subgrid-scale Viscosity Models and Selective Filtering Strategy for Large-eddy Simulations
}

Guillaume Aubard, Pedro Stefanin Volpiani, Xavier Gloerfelt, Jean-Christophe Robinet

\section{- To cite this version:}

Guillaume Aubard, Pedro Stefanin Volpiani, Xavier Gloerfelt, Jean-Christophe Robinet. Comparison of Subgrid-scale Viscosity Models and Selective Filtering Strategy for Large-eddy Simulations. Flow, Turbulence and Combustion, 2013, 91 (3), pp.497-518. 10.1007/s10494-013-9485-5 . hal-01069582

\section{HAL Id: hal-01069582 https://hal.science/hal-01069582}

Submitted on 29 Sep 2014

HAL is a multi-disciplinary open access archive for the deposit and dissemination of scientific research documents, whether they are published or not. The documents may come from teaching and research institutions in France or abroad, or from public or private research centers.
L'archive ouverte pluridisciplinaire HAL, est destinée au dépôt et à la diffusion de documents scientifiques de niveau recherche, publiés ou non, émanant des établissements d'enseignement et de recherche français ou étrangers, des laboratoires publics ou privés. 


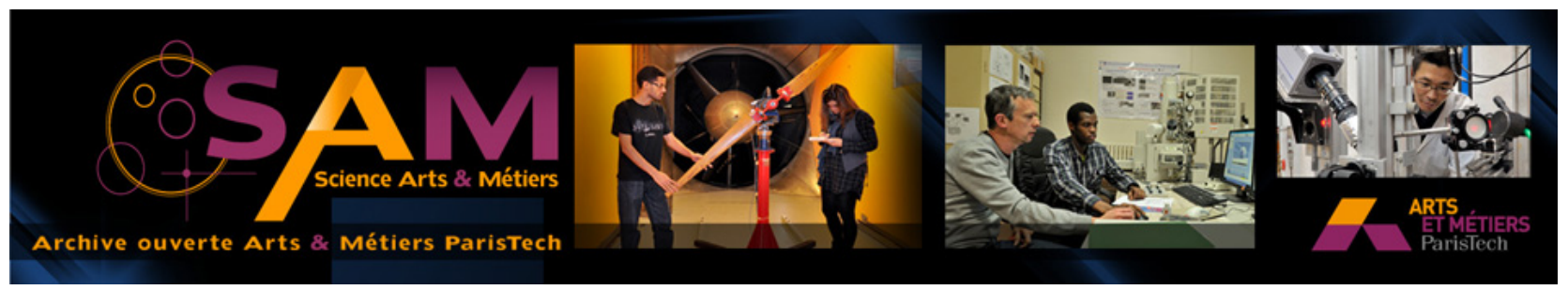

\section{Science Arts \& Métiers (SAM)}

is an open access repository that collects the work of Arts et Métiers ParisTech researchers and makes it freely available over the web where possible.

This is an author-deposited version published in: http://sam.ensam.eu

Handle ID: .http://hdl.handle.net/10985/8637

\section{To cite this version :}

Guillaume AUBARD, Pedro STEFANIN VOLPIANI, Xavier GLOERFELT, Jean-Christophe ROBINET - Comparison of Subgrid-scale Viscosity Models and Selective Filtering Strategy for Large-eddy Simulations - Flow, Turbulence and Combustion - Vol. 91, n³, p.497-518 - 2013 


\title{
Comparison of subgrid-scale viscosity models and selective filtering strategy for large-eddy simulations
}

\author{
G. Aubard · P. Stefanin Volpiani · X. Gloerfelt . \\ J.-C. Robinet
}

Received: date / Accepted: date

\begin{abstract}
Explicitly filtered large-eddy simulations (LES), combining high-accuracy schemes with the use of a selective filtering without adding an explicit subgrid-scales (SGS) model, are carried out for the Taylor-Green-vortex and the supersonic-boundary-layer cases. First, the present approach is validated against direct numerical simulation (DNS) results. Subsequently, several SGS models are implemented in order to investigate if they can improve the initial filter-based methodology. It is shown that the most accurate results are obtained when the filtering is used alone as an implicit model, and for a minimal cost. Moreover, the tests for the Taylor-Green vortex indicate that the discretization error from the numerical methods, notably the dissipation error from the high-order filtering, can have a greater influence than the SGS models.
\end{abstract}

Keywords Large-eddy simulation · Explicit filtering · Taylor-Green vortex · Supersonic boundary layer

\section{Introduction}

In large-eddy simulation (LES), only the most significant scales are discretized by the grid, and the effect of the lacking small scales must be modelled. The separation of large and small scales is done by applying a low-pass filter to the Navier-Stokes equations [1]. A quantity $f$ is decomposed in $\bar{f}+f^{\prime}$, where $\bar{f}$ denotes the resolved filtered part and $f^{\prime}$ the unknown subfilter part. Unclosed terms, such as the subfilter-scale stress tensor, appear in the equations, and can be modelled using a subgrid-scale (SGS) model based on information from the resolved scales.

In traditional LES approaches, the width and the shape of the filter are not explicitely defined and the particular low-pass filtering due to the grid size and the discretization schemes are not taken into account. For example, the filtered quantities are not the same if the convolution with the filter is provided by the grid cutoff (tophat filter of width $\Delta x$ ), or by the projection of a finite number of modes in spectral domain (perfect low-pass cutoff). When

G. Aubard · P. Stefanin Volpiani · X. Gloerfelt · J.-C. Robinet

DynFluid Laboratory, Arts et Metiers ParisTech, 75013 Paris, France

E-mail: xavier.gloerfelt@paris.ensam.fr 
a smooth filter is applied in the physical space, the decomposition $f=\bar{f}+f^{\prime}$ does not provide a clear separation between resolved and unresolved scales since $\bar{f}$ and $f^{\prime}$ overlap in the wavenumber space. Second, unless a spectral method is used, the differentiation introduces an additional spectral truncation of $\bar{f}$. When the underlying discretisation operators play the role of filters, as in implicitly-filtered LES or monotonically-integrated LES (MILES), solutions can be very sensitive to the mesh resolution. Meyers and Sagaut [2] show for instance that better results for wall shear stress in a turbulent channel flow can be obtained by coarsening the grid. Bose et al. [3] or Radhakrishnan \& Bellan [4] demonstrate that the use of an explicit-filter LES allows consistent grid-refinement results. The filter plays a key role in explicitly-filtered LES [5,6]. Since the SGS model commonly requires informations from the smallest resolved scales, it is essential to maintain a high accuracy of numerical schemes near the filter cutoff. The strong interaction between numerical errors and SGS models is well recognised since the studies of Ghosal [7] or Kravchenko and Moin [8]. Even with high-order schemes the subgrid contribution is dominated by numerical errors in the high-wavenumber range.

The SGS models were first derived in the framework of spectral methods, avoiding this complex interaction. The traditional SGS models can be classified into three basic categories: the eddy viscosity model, the similarity model and the mixed model. The eddyviscosity models, first suggested by Smagorinsky [9], based on the Boussinesq approximation in which the SGS Reynolds stresses are related to the strain rate tensor of the resolved field through a viscosity $v_{t}$. Many improvements of the original Smagorinsky model (SM) have been proposed. One of them is the dynamic Smagorinsky model (DSM [10]) which relies on the use of a test filter and on the Germano identity to adapt dynamically the value of $v_{t}$, in order to take into account the presence of solid walls, of laminar flow regions, or of the backscatter effects. However, all eddy-viscosity models suffer from the same limitations. The expression of the SGS tensor being similar to the viscous stress tensor, it acts on all the resolved scales and the effective Reynolds number is artificially decreased [11]. The scale similarity models (SS), proposed by Bardina et al. [12] based on the fact that the largest unresolved scales interact with the smallest resolved scales, do not suffer from this drawback but they cannot reproduce the phases of the complex nonlinear interactions arising at smaller scales, and are therefore underdissipative. The concept of mixed models has then emerged [12], combining the good behaviour of SS and the dissipative nature of eddy-viscosity models such as SM, for stabilization reasons. An other promising LES strategy is to use a scale decomposition motivated by the observation that the effect of SGS on the resolved scales is limited to interactions with scales smaller by a factor of 2 than the smallest resolved scales. Hughes et al. [13] perform a separation ab initio into three types of scales: large, small, and unresolved. This method is referred to as multiscale model, noted MSM or MDSM, depending if a SM or a DSM is applied solely to the small resolved scales, respectively.

In the past few years, a number of new models were proposed that cannot follow this classification. An approach consists in mimicking the dissipative mechanism of the subgrid scales through a regularization procedure. In the ADM model of Stolz et al. [14,15], the deconvolution is underdissipative, and a regularization is obtained by substracting a relaxation term. Gullbrand \& Chow [16] use the ADM deconvolution to reconstruct the velocity after explicit filtering, and add a DSM procedure to achieve sufficient regularization. Several authors propose the use of hyperviscosity [17-19] or spectral vanishing viscosity [20] concepts to provide sufficient dissipation at the smallest scales without recourse to an explicit model. The regularization can also originate directly from the numerical procedure, as in MILES, where the dissipative properties of the numerical scheme constitutes an implicit 
model $[21,22]$. In the truncated Navier-Stokes approach (TNS), proposed by Domaradzki et al. [23-25], the filter is used to damp out the high modes. The interactions between the large and small scales provide necessary dissipation at low modes. Following this idea, a methodology combining low-dissipation schemes with the use a high-order filtering has been developed recently by Stolz et al. [26], Rizzetta et al. [27], or Bogey \& Bailly [28, 29]. Similarly, the present LES strategy combines a finite-difference scheme with good spectral properties with the use of a selective filtering, which has many advantages. It prevents aliasing error and removes grid-to-grid oscillations. It also provides a smooth defiltering by removing the fluctuations at wavenumbers greater than the finite-difference scheme resolvability. As demonstrated by Mathew et al. [30], the effect of ADM is globally similar to the convolution with an explicit filter. In our case, the high-order filter dissipates small scales with a reduced wavenumber $k \Delta x$ greater than $\pi / 2$, which is also the resolvability limit of the finite-difference scheme. Lower scales are almost not affected and are temporally and spatially well resolved, so they do not need to be deconvolved. Moreover, the selective filtering induces a regularization similar to the relaxation term in the ADM model. The effect of SGS motions is taken into account implicitly in the high-wavenumber range thanks to the smooth truncations of the filter and finite-difference schemes in the wavenumber space. That is why Visbal et al. [31] include this kind of approaches in the implicit LES (ILES) category. In the following, the strategy is referred to as RT (for Regularization Term).

The explicit-filter strategy can be used alone as in the RT strategy, or in conjunction with a SGS model. The aim of the paper is to address the effect of adding an explicit SGS model to an explicitly-filtered LES. As pointed out by Berland et al. [32], a systematic assessment of the influence of the filtering procedure parameters, which are the filter shape (determined by the filter-order) and the filtering strength, is still needed. More generally, the relative roles played by the SGS model and the dissipation introduced by the numerical procedure is still unclear.

The paper is organised as follows. First the numerical methodology RT and the different SGS models tested are introduced. Then, the Taylor-Green Vortex case and the supersonic boundary layer case are considered. For both test cases, a mesh convergence is performed up to DNS and the influence of several SGS models is investigated using a relatively coarse mesh. Finally concluding remarks are drawn.

\section{Numerical methods}

\subsection{Governing equations}

The LES is based on the filtered compressible Navier Stokes equations, which can be written in the following conservative form (following Vreman [33]):

$$
\begin{gathered}
\frac{\partial \bar{\rho}}{\partial t}+\frac{\partial\left(\bar{\rho} \tilde{u}_{j}\right)}{\partial x_{j}}=0 \\
\frac{\partial\left(\bar{\rho} \widetilde{u}_{i}\right)}{\partial t}+\frac{\partial\left(\bar{\rho} \widetilde{u_{i}} \widetilde{u}_{j}\right)}{\partial x_{j}}+\frac{\partial \bar{p}}{\partial x_{i}}-\frac{\partial \breve{\sigma}_{i j}}{\partial x_{j}}=-\frac{\partial\left(\bar{\rho} \tau_{i j}\right)}{\partial x_{j}}+\mathscr{R}_{i} \\
\frac{\partial(\bar{\rho} \breve{E})}{\partial t}+\frac{\partial\left((\bar{\rho} \breve{E}+\bar{p}) \widetilde{u}_{j}\right)}{\partial x_{j}}-\frac{\partial\left(\breve{\sigma}_{i j} \widetilde{u}_{i}\right)}{\partial x_{j}}+\frac{\partial \breve{q}_{j}}{\partial x_{j}}=-\frac{\partial\left(\tau_{i j} \widetilde{u}_{i}\right)}{\partial x_{j}}+\mathscr{R}_{e}
\end{gathered}
$$

where $\rho$ represents the density, $u_{i}$ the velocity components and $p$ the pressure. 
The overbar denotes a filtered quantity and the tilde denotes a Favre-filtered quantity. For instance, the Favre-filtered velocity is defined by $\widetilde{u}_{i}=\overline{\rho u_{i}} / \bar{\rho}$. The filtered total energy density is $\bar{\rho} \breve{E}=\bar{p} /(\gamma-1)+\bar{\rho} \widetilde{u}_{i} \widetilde{u}_{i} / 2$ for a perfect gas, where $\gamma$ is the specific heat ratio. The viscous stress tensor is defined by $\breve{\sigma}_{i j}=2 \widetilde{\mu} \breve{S}_{i j}$, where $\breve{S}_{i j}=\left(\partial \widetilde{u}_{i} / \partial x_{j}+\right.$ $\left.\partial \widetilde{u_{j}} / \partial x_{i}\right) / 2-\left(\delta_{i j} \partial \widetilde{u_{k}} / \partial x_{k}\right) / 3$. The viscosity $\widetilde{\mu}=\mu(\widetilde{T})$ is provided by the Sutherland's law $\widetilde{\mu}=\mu_{0}\left(\widetilde{T} / T_{0}\right)^{3 / 2}\left(T_{0}+110,4\right) /(\widetilde{T}+110,4)$, where $T_{0}=273,14 \mathrm{~K}$ and $\mu_{0}=1,711 \times$ $10^{-5} \mathrm{~kg} \cdot \mathrm{m}^{-1} \cdot \mathrm{s}^{-1}$. The filtered temperature $\widetilde{T}$ is obtained using the state equation $\bar{p}=\bar{\rho} r \widetilde{T}$. The heat flux is given by $\breve{q}_{i}=-\left(\widetilde{\mu} c_{p} / \operatorname{Pr}\right)\left(\partial \widetilde{T} / \partial x_{i}\right)$, where $c_{p}$ is the specific heat at constant pressure and $\mathrm{Pr}$ is the Prandtl number. The filtering of the Navier-Stokes equations makes the subgrid terms appear in the right hand side of equations (2) and (3). The most important term contains the SGS turbulent stress tensor defined by $\tau_{i j}=\left(\widetilde{u_{i} u_{j}}-\widetilde{u_{i}} \widetilde{u_{j}}\right)$. The other terms are included in $\mathscr{R}_{i}$ and $\mathscr{R}_{e}$. According to Vreman [34], the quantity $\mathscr{R}_{i}=\partial\left(\bar{\sigma}_{i j}-\breve{\sigma}_{i j}\right) / \partial x_{j}$ can be neglected. The terms that compose the quantity $\mathscr{R}_{e}$ are described in Vreman [34].

\subsection{Numerical strategy}

The convective terms are integrated in time using an explicit low-storage six-substep RungeKutta scheme optimised in the wavenumber space by Bogey \& Bailly [35]. Because of their slower time evolution, the viscous terms are only integrated in the last substep. The gradients are solved on a Cartesian grid by using finite differences on a fourth-order 11-point stencil scheme optimised by Bogey \& Bailly [35] for the convective fluxes, and standard fourthorder finite differences for the viscous and heat fluxes. The non-uniformity in the mesh sizes is taken into account by a suitable 1-D coordinate transform. For instance stretching rate of 1.5 to $2 \%$ are used in the wall-normal direction for the boundary-layer simulations. As part of the algorithm, a selective filtering is incorporated in each direction to eliminate high-wavenumber oscillations. We use an optimised sixth-order centered filter built on an 11-point stencil [36]. For instance in the direction $x$, a filtered quantity $\bar{f}^{f}$ is computed as

$$
\bar{f}^{f}\left(x_{0}\right)=\bar{f}\left(x_{0}\right)-\chi D_{f}\left(x_{0}\right) \text { with } D_{f}\left(x_{0}\right)=\sum_{m=-5}^{5} d_{m}^{11} \bar{f}\left(x_{0}+m \Delta x\right)
$$

with a fixed amplitude $\chi(0<\chi<1)$. The filter has symmetric coefficients $d_{m}^{11}$, so that it is non-dispersive. The properties in the Fourier space of the finite differences and of the filter are depicted in Fig.1(a) and (b). The dispersion of the finite differences is maintained at a very low level up to the limit of resolvability $k \Delta x=\pi / 2$, corresponding to four points per wavelength. The filter scheme has a slightly lower limit of accuracy. The larger scales that are accurately discretized are affected in a negligible manner by the filtering, whereas the smaller scales, badly calculated, are damped out.

\subsection{Subgrid-scale models}

The first class of models tested is based on the Smagorinsky model. For the classical Smagorinsky model (SM), a SGS eddy viscosity is defined by $v_{t}=C_{S}^{2} \bar{\Delta}^{2}\left(2 \breve{S}_{i j} \breve{S}_{i j}\right)^{1 / 2}$, where $\bar{\Delta}=$ $(\Delta x \Delta y \Delta z)^{1 / 3}$ and $C_{S}$ is the Smagorinsky constant. Moreover, the isotropic part of the SGS tensor $\tau_{i j}$ is modelled, according to Yoshizawa [37], by $\tau_{k k}=2 C_{I} \bar{\rho} \bar{\Delta}^{2}\left(2 \breve{S}_{i j} \breve{S}_{i j}\right)^{1 / 2}$, where 

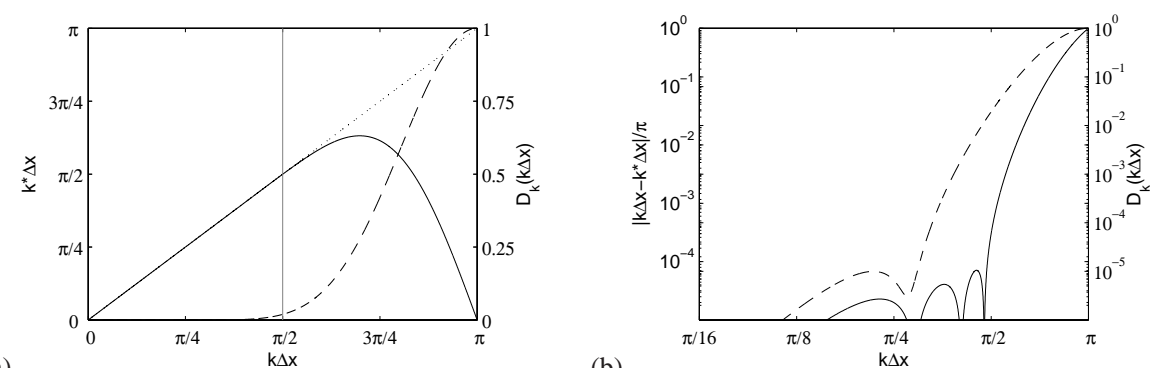

(a)

Fig. 1 Fourier analyses of central finite-difference and filter schemes. (a) The effective wavenumber $k^{*} \Delta x$ of the 11-point finite-difference scheme ( - ) and the damping function $D_{k}(k \Delta x)$ of the 11-point filter ( - - - ) are plotted as function of the reduced wavenumber $k \Delta x$ with scales on the left and on the right, respectively. The dotted line represents the exact relationship for the discretization scheme and the vertical gray line marks the limit of resolvability $k \Delta x \approx \pi / 2$. (b) Logarithmic scales are proposed for the dispersion error of finite differences ( - ) with scale on the left axis and for the dissipation error of the filter ( - - ) with scale on the right axis.

$C_{I}=0.066$ [38]. Finally the quantity $\mathscr{R}_{e}$ of equation (3) is modelled, according to Vreman [34], by $\mathscr{R}_{e}=-\frac{\partial}{\partial x_{j}}\left(\frac{c_{p} \bar{\rho} v_{t}}{\operatorname{Pr}_{t}} \frac{\partial \tilde{T}}{\partial x_{j}}\right)$ where the turbulent Prandtl number $\operatorname{Pr}_{t}=0.6$ is fixed. A van Driest damping function is added for the wall-bounded case. Secondly, the dynamic version (DSM) is also implemented, where the test filter is a Laplacian, and for the inhomogeneous case the dynamic constant is filtered with a Laplacian to avoid too fast variations. Third, the multiscale model in conjunction with SM (MSM) or DSM (MDSM) is tested. The scale separation is performed by an 11-point filter with a cutoff at $k \Delta x=\pi / 3$ [35]. In the small-small version (MSM-ss or MDSM-ss), both the SGS tensor and the eddy viscosity are based on the filtered rate of strain tensor, whereas in the large-small version (MSM-ls or MDSM-ls), the SGS tensor alone is based on the filtered rate of strain tensor. Finally, the class of mixed models combining Scale Similarity (SS) and SM (SM/SS), DSM (DSM/SS) or RT (RT/SS) is implemented. The scale similarity term is $\widehat{\widetilde{u_{i}} \widetilde{u}_{j}}-\widehat{\tilde{u}_{i}} \widehat{\tilde{u}_{j}}$ [39], where $\widehat{\text { is an }}$ 11 -point test-filter with a cutoff at $k \Delta x=\pi / 3$ [35]. In this study, we restrict the number of models to the most representative.

We recall that for the RT procedure, no explicit SGS model is added. The required regularization is provided by the filtering procedure, already used as the numerical dissipation of the spatial scheme. That is why Visbal et al. [40] include this kind of approaches in the implicit LES (ILES) category.

\section{Taylor-Green Vortex}

The first test case considered is the Taylor-Green Vortex (TGV). It is a fundamental case used as prototype for vortex stretching and production of small-scale eddies (see figure 2), and therefore allows the study of the dynamics of transition to turbulence. The configuration involves triple-periodic boundary conditions enforced on a cubical domain box side length 
$2 \pi$. The flow is initialized as:

$$
\begin{aligned}
u_{0}(\mathbf{x}) & =U_{\infty} \sin (x) \cos (y) \cos (z) \\
v_{0}(\mathbf{x}) & =-U_{\infty} \cos (x) \sin (y) \cos (z) \\
w_{0}(\mathbf{x}) & =0 \\
p_{0}(\mathbf{x}) & =p_{\infty}+\frac{q_{\infty}}{16}[\cos (2 z)+2][\cos (2 x)+\cos (2 y)]
\end{aligned}
$$

where $q_{\infty}=\rho_{\infty} U_{\infty}^{2}$. We choose $U_{\infty}=1, p_{\infty}=100$ and $\rho_{\infty}=1$, corresponding to a Mach number $\mathrm{M}=0.085$. The Reynolds number is $\mathrm{Re}=1600$, as in the study of Hickel et al. [41], which is large enough to have a rapid transition into small-scales turbulence. The TGV configuration has been used by several authors to assess LES closures [42-44]. In particular, in the recent study of Fauconnier et al. [45], the filter-based strategy is investigated within an incompressible spectral solver at $\mathrm{Re}=3000$. Results are obtained for the influence of the filter's order and amplitude without discretization errors, and are thus of particular interest for comparison.

First, a grid convergence study is performed using RT strategy using $32^{3}$ to $256^{3}$ points, with $4^{\text {th }}$-order 11-point optimised scheme and $6^{\text {th }}$-order 11-point optimised filtering applied with an amplitude $\chi=0.1$. The results for the kinetic energy $k$ and for the kinetic energy dissipation $\varepsilon=-d k / d t$ are compared in Fig. 3 with the incompressible DNS of Brachet et al. [46] using $256^{3}$ points and spectral schemes. The most refined case is in very good agreement with the DNS reference, which shows the ability of the RT strategy to reproduce the dynamics of the TGV case. Satisfactory results are obtained with the $64^{3}$ mesh since both the transition region and the turbulence production peak are close, but not superposed, to the DNS reference. In particular, in all simulations on a $64^{3}$ grid, the sharp peak near $t=9$ in the dissipation history appears as spread and even splitted. Another event which is difficult to capture corresponds to the instant $t=5$ where stretched vortex tubes break down into small-scales turbulence. An undue inflection often appears at this time in the dissipation curves. In the following, the $64^{3}$ mesh is used to conduct the LES comparisons.

The influence of the RT strategy parameters is first evaluated, namely the numerical scheme, the filter stencil and its amplitude $\chi$. If not specified, the optimised fourth-order 11-point stencil spatial scheme [35] and the optimised sixth-order 11-point stencil filter [36] are used with a filtering amplitude $\chi=0.1$.

The first investigation concerns the spatial discretization scheme. Two families of central finite-difference schemes are tested, namely standard versions, where the coefficients for a stencil of $2 N+1$ points are obtained by cancelling terms up to order $2 N$ in Taylor's expansions, and optimised schemes, where certain coefficients are used to minimize the dispersion error in the wavenumber space. Figure 4(a) shows the influence of the order of the standard finite-difference schemes. The dissipation curve of the $2^{\text {nd }}$-order scheme experiences strong oscillations particularly near the transition time and near the turbulence production peak. The phase errors thus severely alter the slope of the kinetic energy decrease. Weak oscillations are also present with the $4^{\text {th }}$-order scheme. The curves obtained beyond the $6^{\text {th }}$-order are rather similar, showing that the quality of the solution becomes independent of the spatial scheme order. Figure 4(b) shows the influence of the spatial scheme stencil for optimised finite differences, referred to as dispersion-relation-preserving (DRP) schemes. The curves are almost superposed for the three stencil tested, showing that, simi- 

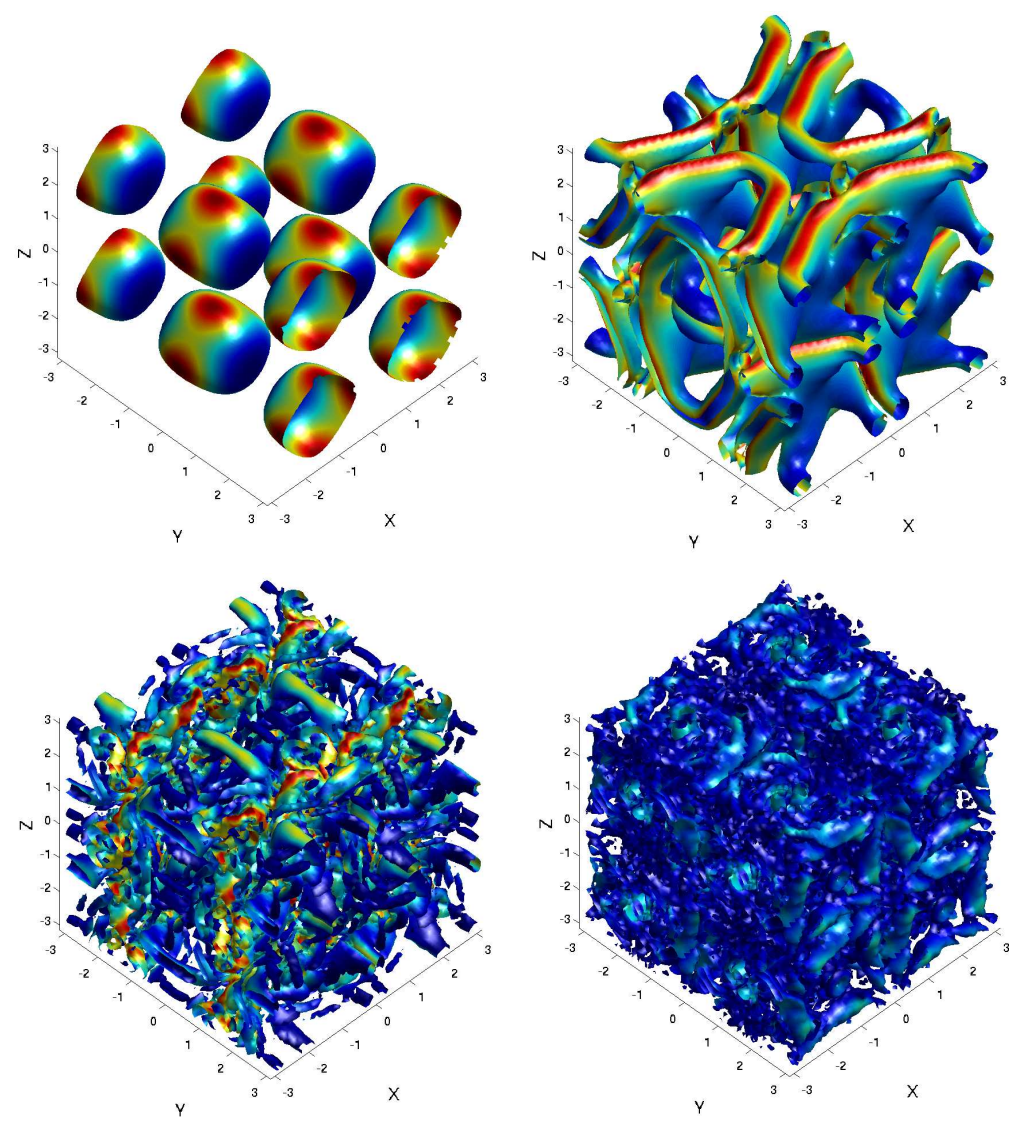

Fig. 2 Iso-surfaces of the Q-criterion colored by the kinetic energy, for the LES of the Taylor-Green case with a $64^{3}$ mesh. Four successive non-dimensionalised times are presented, corresponding to (a) the initialisation $(t=0)$, (b) the laminar region ( $t=3.75)$, (c) the transition region $(t=9)$, and (d) the decaying small-scales turbulence region $(t=15)$.

larly to standard schemes, little influence of the stencil size is found beyond a 7-point stencil.

Figures 5(a) and 5(b) show the influence of the filter order in the RT method for standard filters and for optimised filters respectively. Standard centered filter have symmetric coefficients on a $2 N+1$ point stencil obtained by cancelling terms up to order $2 N$ in a Taylorseries expansion. Optimised filter use one or two coefficients to minimize the dissipation error in the wavenumber space. The formal order is thus reduced but the resolvability can be enhanced. Concerning the standard filters, $4^{\text {th }}$-order and $6^{\text {th }}$-order filters are overdissipative in the transition region (for $t \simeq 5$ ). For filters beyond the $8^{\text {th }}$-order, the influence is weak in good agreement with the results of Fauconnier et al. [45]. The behaviour of optimised filters is quite different. The 7-point optimised filter strongly overestimates the dissipation at the beginning of the simulation where the flow is still laminar. The laminar region seems to be very sensitive to the level of dissipation in the numerical method. Due to the optimization, a residual dissipation can be present in the low-wavenumber region (marked by the bounces in a Fourier representation such as Fig.1). The 7-point optimised filter has not 


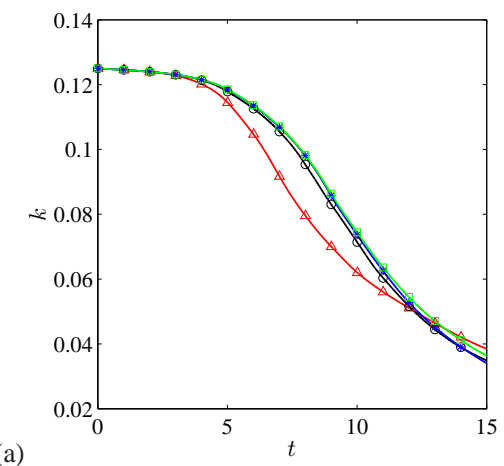

(b)

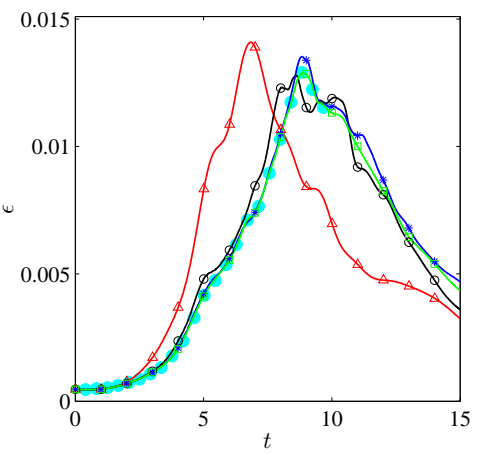

Fig. 3 Influence of the grid for the Taylor-Green vortex. (a) Kinetic energy $k$ and (b) kinetic energy dissipation $\varepsilon$ for: $(\square \square) 32^{3}$; ( $\square$ ) $64^{3} ;(\square+) 128^{3}$; ( $\square$ ) $256^{3} ;(\bullet \bullet \bullet)$ Brachet et al. [46].

(a)

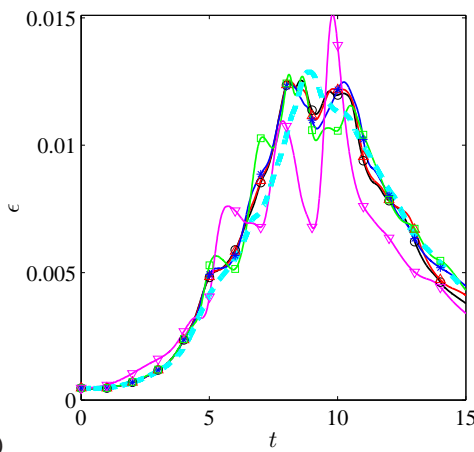

(b)

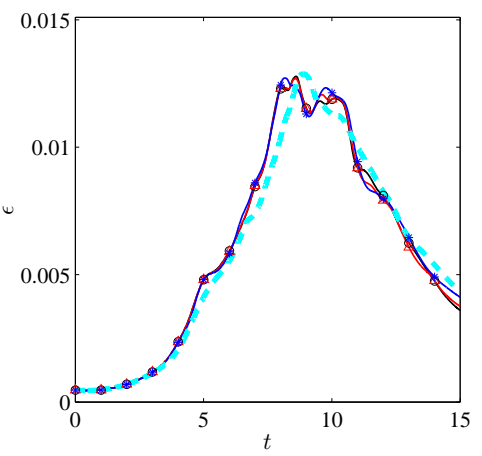

Fig. 4 Influence of the spatial scheme stencil for the Taylor-Green vortex. (a) For standard finite differences. Kinetic energy dissipation $\varepsilon$ for: $(\square \vee) 2^{\text {nd }}$-order scheme; $(\square, \quad) 4^{\text {th }}$-order scheme; ( $\sim$ ) $6^{\text {th }}$-order scheme; ( $\left.\square\right) 8^{\text {th }}$-order scheme; ( $\square$ ) $10^{\text {th }}$-order scheme. (b) For optimised finite differences. Kinetic energy dissipation $\varepsilon$ for: ( $\square$ ) 7-point DRP scheme ([47]); ( $\square$ ) 9-point DRP scheme ([35]); ( $\quad$ ) 11-point DRP scheme ([35]); ( - - ) $256^{3}$ (see Fig.3).

enough degrees of freedom to avoid unduly damping of kinetic energy, resulting in a drift during the laminar phase. Significant improvement is obtained with the 9-point stencil even if a slight overestimation in the transition region is observed. Therefore, contrary to the results obtained for finite differences schemes, a major influence is found up to the $8^{\text {th }}$-order for standard filters or for a stencil of at least 11 points for the optimised filters. These results are consistent with those of Berland et al. [32] on a mixing layer, which show a major influence of the filter shape, directly linked to the filter order. These results show that the accuracy of the LES solution depends significantly on the choice of a sufficiently sharp filter.

Figure 6 shows the influence of the filter amplitude $\chi$ used in the RT methodology . All the curves are rather similar, indicating a weak influence of $\chi$ for both standard (Fig.6(a)) and optimised filters (Fig.6(b)). Small discrepancies are however found around time $t=5$, corresponding to the appearence of small turbulent scales, suggesting a non-negligeable influence of the filter amplitude. Fauconnier et al. [45] also found a weaker influence of the 

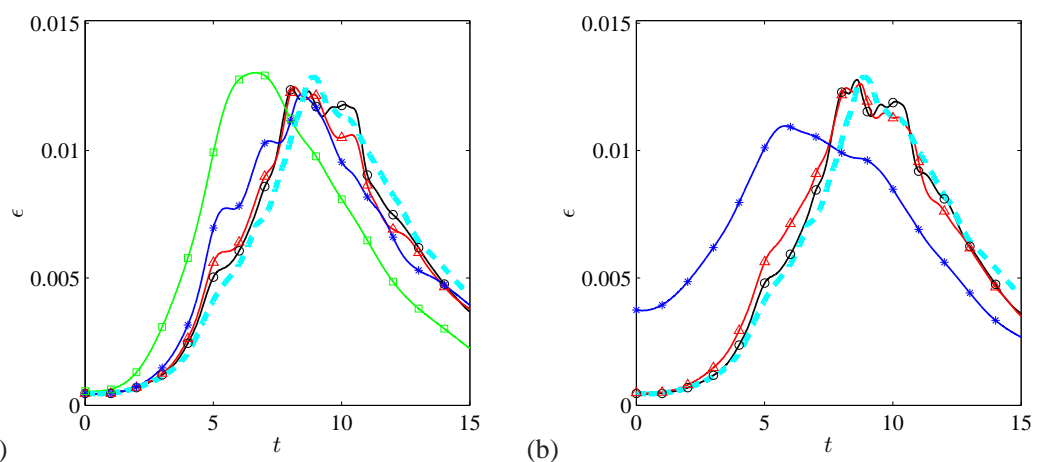

Fig. 5 Influence of the RT methodology filter stencil for the Taylor-Green vortex. (a) For standard filters. Kinetic energy dissipation $\varepsilon$ for: $(\square \square) 4^{\text {th }}$-order; $\left(\square^{-}\right) 6^{\text {th }}$-order; $(\square \square) 8^{\text {th }}$-order; $\left(\square\right.$ ) $10^{\text {th }}$-order. (b) For optimised filters. Kinetic energy dissipation $\varepsilon$ for: ( $\left(-\frac{\pi}{*}\right) 7$-point DRP filter ([35]); ( $\square$ ) 9-point DRP filter ([35]); ( $\square$ ) 11-point DRP filter ([36]); ( $=-$ ) $256^{3}$ (see Fig.3).
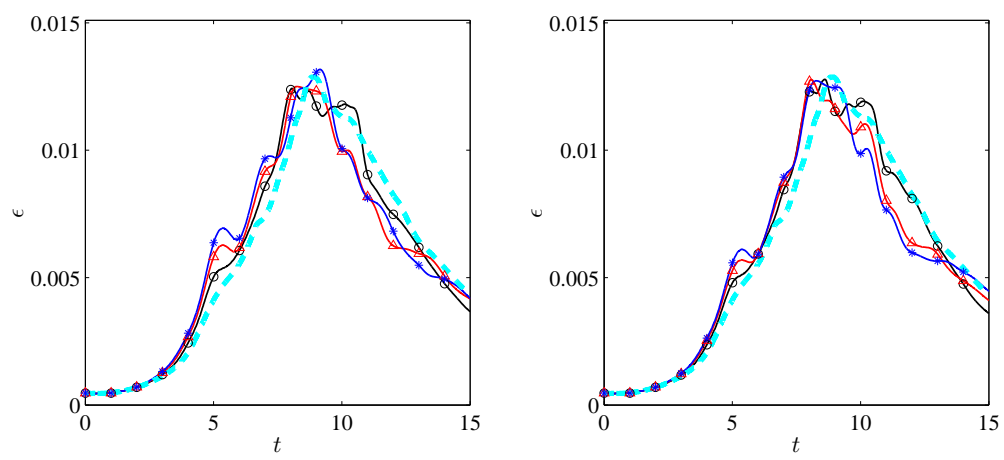

Fig. 6 Influence of the filter amplitude $\chi$ in the RT method for the Taylor-Green vortex. (a) For standard $10^{\text {th }}$-order filter; (b) For optimised $6^{\text {th }}$-order 11 -point filter; Kinetic energy dissipation $\varepsilon$ for: $(\square-\chi)=0.1$; $\left(\square\right.$, ) $\chi=0.4 ;(\square) \chi=0.8 ;(-\square-) 256^{3}$ (see Fig.3).

filtering strength with respect to its order. They have shown that the amplitude can be related to the filtering frequency or to the relaxation time as introduced in the regularization term of Stolz et al. [14].

The comparison of several SGS models is presented in Fig.7 and 8. In this series, the 11-point optimised finite-difference and filter schemes are used with $\chi=0.1$. Figure 7 (a) focuses on SM-type models. The Smagorinsky model, even in its dynamic form, appears to be too dissipative and describes poorly the laminar-turbulent transition region. The dissipation effect is triggered to early resulting in a shift of the peak dissipation toward earlier times. The most dissipative case is the SM with $C_{S}=0.18$, and shows an underestimation of the turbulence production peak. The use of multiscale models (Fig.7(b)) allows a significant improvement of the quality of the solution, but shows results similar to those obtained with the RT strategy alone (see Fig.5(b)). Similar conclusions are obtained in Fig.8(a) with the use of mixed models. Figure 8 (b) gathers some of the most representative models. Results similar to those obtained with the RT methodology can be obtained with the use of multi- 


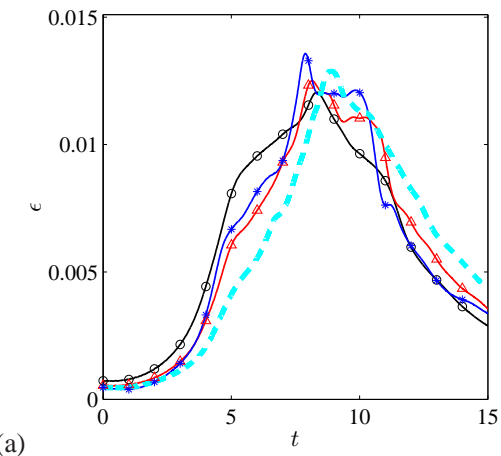

(b)

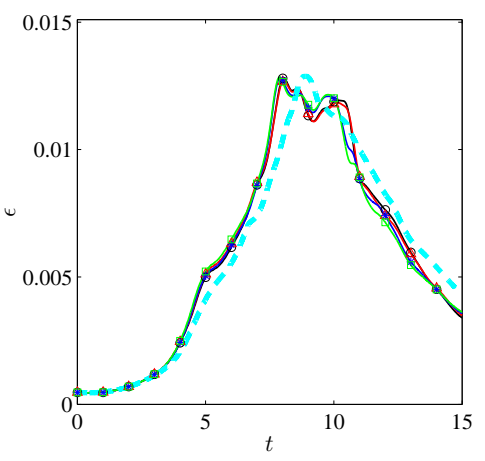

Fig. 7 Influence of the SGS model for the Taylor-Green vortex. (a) Smagorinsky-type models. Kinetic energy dissipation $\varepsilon$ for: ( $\square$ ) SM with $C_{S}=0.18$; ( $\square$ ) SM with $C_{S}=0.1$; ( $\square$ ) DSM. (b) Multi-scale Smagorinsky models. Kinetic energy dissipation $\varepsilon$ for: $(\square)$ MSM-ss; ( $\square$ ) MSM-ls; ( $\div$ ) MDSM-ss; ( $\square$ ) MDSM-ls. ( $\square-$ ) $256^{3}$ (see Fig.3).

(a)

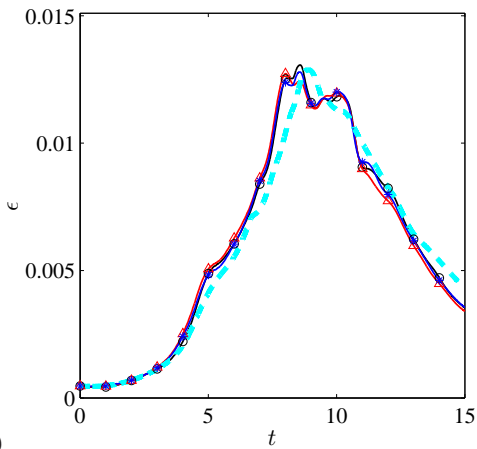

(b)

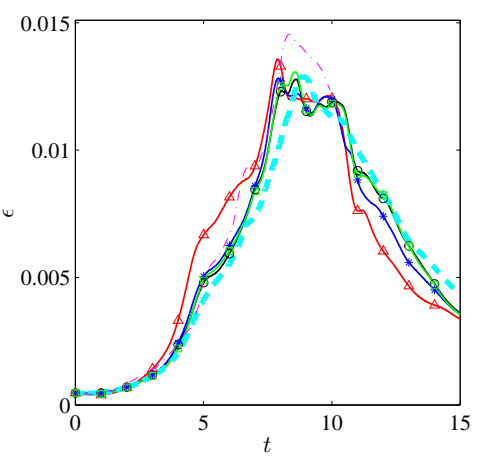

Fig. 8 Influence of the SGS model for the Taylor-Green vortex. (a) Mixed models. Kinetic energy dissipation $\varepsilon$ for: $(-\square)$ DSM/SS; ( $\square$ ) MSM-ss/SS; ( - ) RT/SS. (b) Main models. Kinetic energy dissipation $\varepsilon$ for: ( $\square$ ) RT with $\chi=0.1$; ( $\square$ ) DSM; ( $\square$ ) MDSM-ss; ( $\square$ ) DMM; $(-\cdots)$ ALDM [41]; (- -- ) $256^{3}$ (see Fig.3).

scale or mixed models but for a higher cost. No improvement of the initial RT stategy is observed. Figure 8(b) also indicates a better agreement with the DNS than the recent elaborated ALDM (Adaptive Local Deconvolution Method) model of Hickel et al. [41] using 64 ${ }^{3}$ points, probably due to their discretization scheme. This particular result confirms that the numerical errors can dominate the role of the SGS-model.

\section{Supersonic turbulent boundary layer}

\subsection{DNS simulation and mesh convergence}

The spatial development of a supersonic turbulent boundary layer is considered. The freestream Mach number is $\mathrm{M}_{\infty}=2.25$ and the Reynolds number $\operatorname{Re}_{\theta}=U_{\infty} \theta / \nu$ based on the momentum thickness is 3000 at the location where the data are extracted. The free-stream 
temperature and pressure are respectively $T_{\infty}=169.44 \mathrm{~K}$ and $p_{\infty}=23999 \mathrm{~Pa}$. The inflow condition is obtained by adding perturbations on a laminar boundary layer velocity profile that is deformed near the wall to exhibit an inflection point. Inflectional Kelvin-Helmholtz-type instabilities will then trigger a relatively rapid transition toward a turbulent state. The idea is to use a very long inlet domain to become independent of the inflow. On the wall boundary, the no-slip conditions $u_{i}=0$ are imposed, with $\partial p / \partial n=0$ for the Eulerian part, where $n$ is the direction normal to the solid surface. The wall temperature is calculated with the adiabatic condition, and the density can be deduced using ideal gas law. The finite-difference stencil for the convective terms is progressively reduced down to the second order as the wall is approached and non-centered 11-point stencils are used for the filtering operation. The values of the coefficients of the non-centered filters can be found in Berland et al. [48]. At the upstream and upper boundaries of the computational domain, nonreflecting characteristic boundary conditions [49] are applied. A sponge zone [50] is furthermore added at the downstream end of the domain so that unhindered passage of aerodynamic perturbations is possible without the generation of spurious acoustic waves from the subsonic zone. A threedimensional planar domain is considered with periodic boundary conditions in the spanwise direction. First, a DNS simulation is performed to be used as a reference. The mesh used and the wall-unit resolution are given in Table 1 . The amplitude of the filtering is fixed at a low value $\chi=0.2$.

\begin{tabular}{|c|c|c|c|c|c|c|c|c|c|}
\hline Grid levels & $N_{x}$ & $N_{y}$ & $N_{z}$ & $\Delta x$ & $\Delta y$ & $\Delta z$ & $\Delta x^{+}$ & $\Delta y^{+}$ & $\Delta z^{+}$ \\
\hline DNS & 2560 & 200 & 300 & $3.2 \times 10^{-5}$ & $2.1 \times 10^{-6}$ & $1.7 \times 10^{-5}$ & 13 & 0.9 & 7 \\
\hline LES 1 & 1280 & 200 & 150 & $3 \Delta x_{D N S}$ & $2 \Delta y_{D N S}$ & $2 \Delta z_{D N S}$ & 40 & 1.8 & 14 \\
\hline LES 2 & 1280 & 180 & 50 & $4 \Delta x_{D N S}$ & $3 \Delta y_{D N S}$ & $3 \Delta z_{D N S}$ & 53 & 2.7 & 21 \\
\hline LES 3 & 1032 & 150 & 38 & $5 \Delta x_{D N S}$ & $4 \Delta y_{D N S}$ & $4 \Delta z_{D N S}$ & 69 & 3.6 & 28 \\
\hline
\end{tabular}

Table 1 Mesh configurations for the DNS and LES simulations.

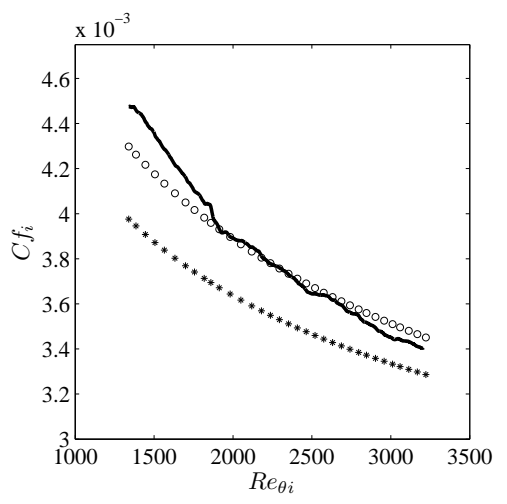

Fig. 9 DNS of the supersonic boundary layer. Incompressible friction coefficient $C f_{i}$ : (-) DNS; (०) Blasius correlation $C f_{B L}[51]$; ( $\star$ Kármán/Schoenherr correlation $C f_{K S}$ [51]. 

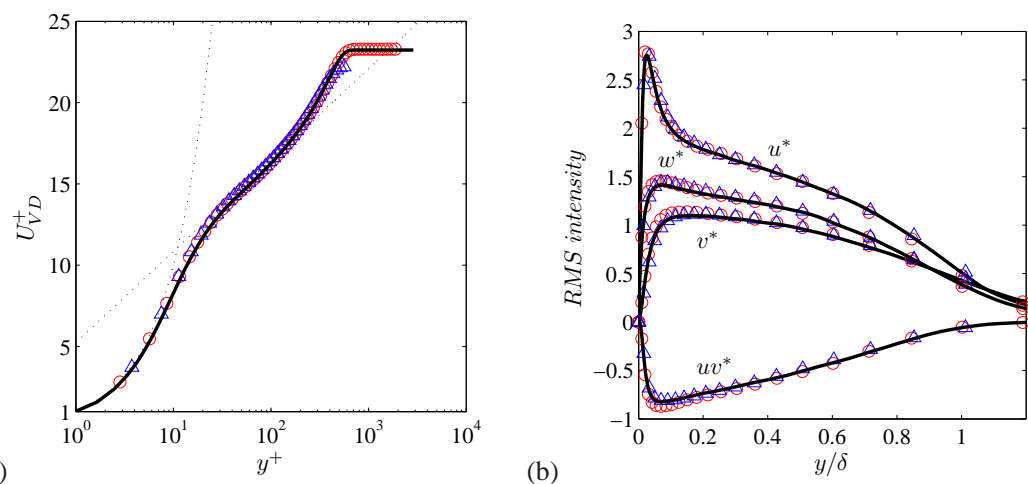

Fig. 10 DNS of the supersonic boundary layer. (a) van Driest transformed mean velocity profiles; (b) density scaled $r m s$ velocity profiles; ( - ) DNS; $(\triangle)$ Pirozzoli \& Bernardini [52] at $M=2.28$ and $\operatorname{Re}_{\theta}=$ 2300; (०) Bernardini \& Pirozzoli [53] at $\mathrm{M}=2$ and $\operatorname{Re}_{\theta}=3000$.

The longitudinal evolution of the friction coefficient is a good indicator of a fully developed turbulent state. The van Driest II transformation of the friction coefficient is compared in Fig.9 with the incompressible Kármán/Schoenherr and Blasius correlations [51]. The incompressible friction coefficient is obtained by $C f_{i}=C f\left(T_{w} / T_{\infty}-1\right) / \arcsin ^{2} \alpha$ with $\alpha=\left(T_{w} / T_{\infty}-1\right) / \sqrt{T_{w} / T_{\infty}\left(T_{w} / T_{\infty}-1\right)}$. The incompressible Reynolds number is obtained by $\operatorname{Re}_{\theta_{i}}=\frac{\mu_{\infty}}{\mu_{w}} \operatorname{Re}_{\theta}$, and the Kármán/Schoenherr $C f_{K S}$ and Blasius $C f_{B L}$ correlations [51] by

$$
C f_{K S}=\frac{1}{17.08 \log \left(\operatorname{Re}_{\theta_{i}}\right)^{2}+25.11 \log \left(\operatorname{Re}_{\theta_{i}}\right)+6.012} \text { and } C f_{B L}=\frac{0.026}{\operatorname{Re}_{\theta_{i}}^{1 / 4}}
$$

Results of Fig.9 show that a fully turbulent state is obtained for $\operatorname{Re}_{\theta_{i}}=2000$, which corresponds to $\operatorname{Re}_{\theta}=2250$. The choice is then made to perform the comparisons with numerical references at $\operatorname{Re}_{\theta}=3000$.

The DNS results in Fig.10 show very good agreement with recent compressible DNS at similar Reynolds and Mach numbers, both for the van Driest mean velocity profile $U_{V D}^{+}$ (Fig.10(a)) and the density scaled rms velocity profiles (Fig.10(b)).

An equation for the turbulent kinetic energy budget in compressible LES based on explicit filtering has been derived in Bogey \& Bailly [55] and is implemented in this study. The filtering process of the density variable is equivalent to the first order explicit integration over the time $\Delta t$ of the operator

$$
D_{f}(\bar{\rho})_{i, j, k}=-\frac{\chi}{\Delta t} \sum_{m=-5}^{5} d_{m}^{11}\left(\bar{\rho}_{i+m, j, k}-\left\langle\bar{\rho}_{i+m, j, k}\right\rangle\right)
$$

where $\langle$.$\rangle is the statistical average and [.] is the Favre average. A similar procedure can be$ used for the filtering of the momentum variables. The term associated with the filtering dissipation is then given by $u_{i}^{\prime} D_{f}\left(\overline{\rho u_{i}}\right)$. The main terms of the budget are successively the production term $\left(-\left\langle\bar{\rho} u_{i}^{\prime} u_{j}^{\prime}\right\rangle \frac{\partial\left[u_{i}\right]}{\partial x_{j}}\right)$, the turbulent transport term $\left(-\frac{1}{2} \frac{\partial}{\partial x_{j}}\left\langle\bar{\rho} u_{i}^{\prime 2} u_{j}^{\prime}\right\rangle-\frac{\partial}{\partial x_{j}}\left\langle p^{\prime} u_{i}^{\prime}\right\rangle\right)$, the viscous diffusion term $\left(\frac{\partial}{\partial x_{j}}\left\langle u_{i}^{\prime} \breve{\sigma}_{i j}\right\rangle\right)$, and the viscous dissipation term $\left(-\left\langle\breve{\sigma}_{i j} \frac{\partial u_{i}^{\prime}}{\partial x_{j}}\right\rangle\right)$. When an explicit SGS is present, its contribution is the SGS dissipation term $\left(-\left\langle\tau_{i j}^{S G S} \frac{\partial u_{i}^{\prime}}{\partial x_{j}}\right\rangle\right)$, 


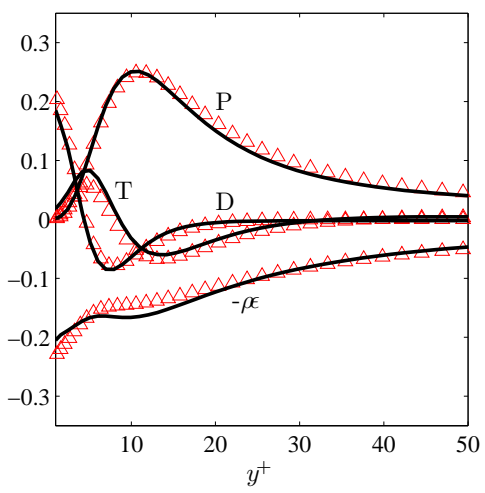

Fig. 11 DNS of the supersonic boundary layer. Turbulent kinetic energy budget: P: production; T: turbulent transport; D: viscous diffusion; $-\rho \varepsilon$ : viscous dissipation; (- ) DNS; $(\triangle)$ Schlatter \& Örlü [54] at $\operatorname{Re}_{\theta}=4060$.

(a)

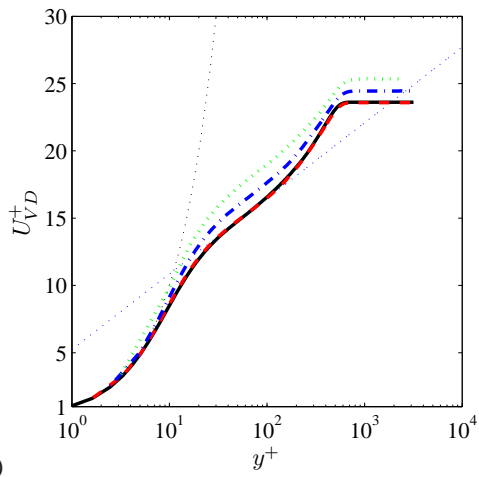

(b)

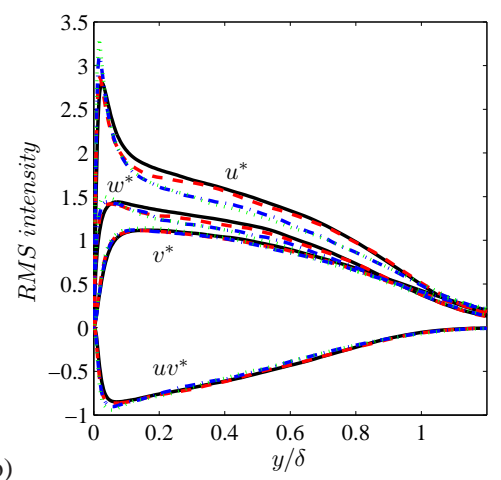

Fig. 12 Grid convergence for the supersonic boundary layer. (a) van Driest transformed mean velocity profiles; (b) density scaled rms velocity profiles; ( - ) DNS; ( - - - ) LES1; ( - - - ) LES2; $(\cdots \cdots)$ LES3.

where $\tau_{i j}^{S G S}$ is calculated from the SGS viscosity $v_{t}$ and the SGS kinetic energy. These terms, normalized by $\rho_{w} u_{\tau}^{4} / v_{w}$, are presented in Fig. 11 for the DNS case. A good agreement with the incompressible DNS of Schlatter \& Örlü [54] is noted, giving good confidence in the numerical strategy.

Three LES simulations using RT strategy are then performed with a progressively coarsened resolution (see Table 1). Figure 12 shows the effect of the mesh coarsening on the van Driest mean velocity profile $U_{V D}^{+}$(Fig.12(a)) and the density scaled $r m s$ velocity profiles (Fig.12(b)). The coarsening leads to an underprediction of the friction velocity, evaluated here using a non-centered $4^{\text {th }}$-order wall-normal derivative of the velocity, and therefore to an overprediction of the law of the wake. An underprediction of the rms velocity profiles is also observed, particularly for the longitudinal velocity, excepted for the near-wall turbulence peak which is overestimated. The effect of coarsening on the turbulent kinetic energy is showed in Fig.13(a). The major effect is an underprediction of the production term, related 

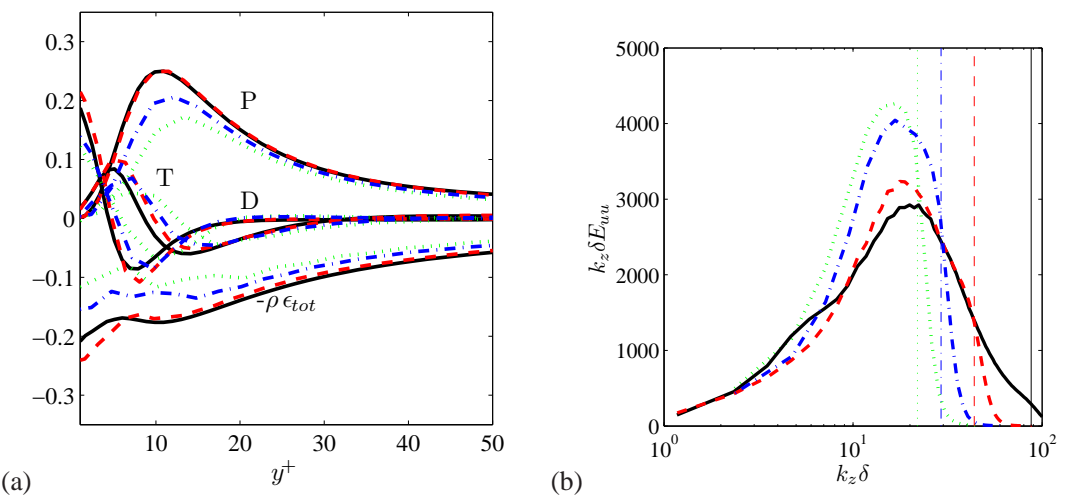

Fig. 13 Grid convergence for the supersonic boundary layer. (a) Turbulent kinetic energy budget: P: production; T: turbulent transport; D: viscous diffusion; $-\rho \varepsilon_{\text {tot }}$ : total dissipation (viscous dissipation + filtering dissipation); (b) Pre-multiplied wavenumber spectra at $y / \delta_{99}=0.03$ for the spanwise direction $k_{z}$;

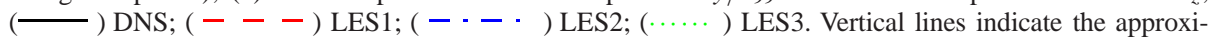
mate cutoff wavenumber for each simulations.

to the underestimation of the Reynolds stresses. A subsequent underprediction of the global dissipation, obtained by summing the viscous and the RT-filtering dissipation, is also observed. A look at the pre-multiplied spanwise wavenumber spectra in Fig.13(b) shows that coarsening the mesh reduces the energy at small scales by degrading the numerical resolution, this energy being transfered to lower wavenumber scales. Note that we have estimated the cutoff wavenumber in the spanwise direction by $2 \pi /(4 \Delta z)$, by considering that roughly 4 points per wavelength are needed by the spatial scheme.

\subsection{Comparison of SGS models}

In this section, the comparison of different SGS models is presented only on the coarsest mesh, referred to as LES 3 in Table 1, but the same analysis on the intermediate mesh, referred to as LES 2 in Table 1, gives similar qualitative results.

Figures 14 and 15 show 2-D maps of the turbulent viscosity $v_{t}$ for some of the tested models. The SGS viscosity is normalized by the viscosity of the fluid $v$ and the normal direction is stretched for clarity reasons. The Smagorinsky model with $C_{S}=0.15$ generates an important turbulent viscosity for both instantaneous and time-averaged fields. As expected, significantly lower levels are obtained with a constant of $C_{S}=0.1$. The dynamic version leads to local values of the turbulent viscosity similar to the case SM with $C_{S}=0.15$ but to timeaveraged values similar to the case $\mathrm{SM}$ with $C_{S}=0.1$. This shows that the dynamic model generates strong gradients of $v_{t}$, and has therefore a high adaptability. The spots of color observed in the case of the dynamic model are the results of the smoothing procedure performed by a Laplacian filter. Much lower values of $v_{t}$ are obtained with the MSM-ss model both for instantaneous and time-averaged fields. The dissipation introduced by the present SGS models are in good agreement with the results obtained on the TGV case. Moreover, significant turbulent viscosity levels are observed near the location of the inflow discontinuity shock, particularly for the DSM model. This shows the potential of SGS modelisation 


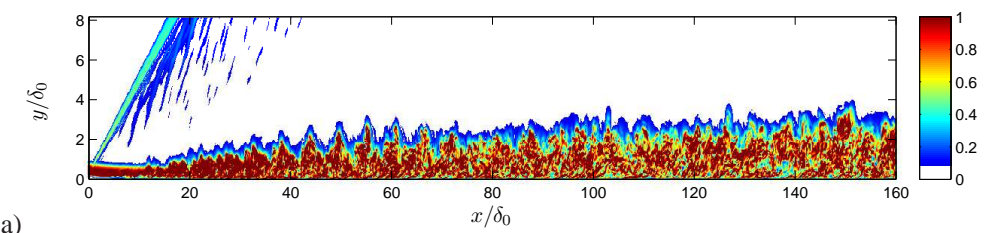

(a)

$x / \delta_{0}$

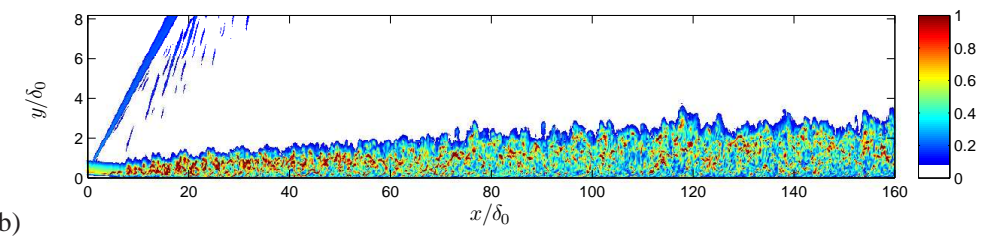

(b)

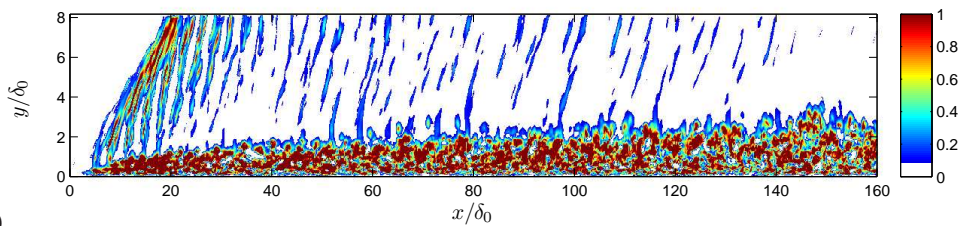

(c)

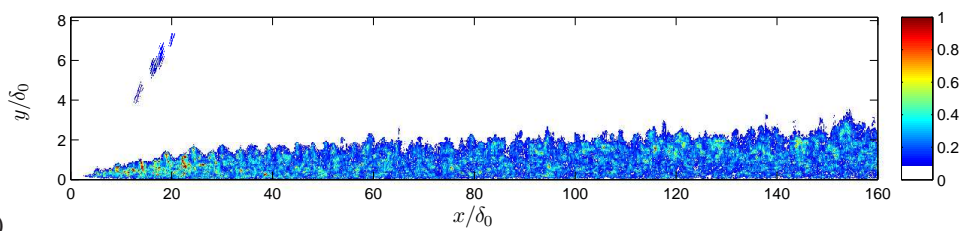

Fig. 14 Influence of the SGS model for the supersonic boundary layer. Colormaps of instantaneous normalized eddy viscosity $v_{t} / v$ (levels between 0 and 1); (a) SM with $C_{S}=0.15$; (b) SM with $C_{S}=0.1$; (c) DSM; (d) MSM-ss. $\delta_{0}$ is the initial boundary layer thickness.

for shock-capturing procedures [56].

Figure 16(a) shows the influence of the models on the rms velocity profiles. All the models tested give similar results and overestimate the near-wall turbulent peak for the longitudinal velocity. The location of the peak is also in very good agreement with the DNS results. The peak locations for the SM models and particularly the SM model with $C_{S}=0.15$ are however found slighty farther from the wall, indicating a diminution of the effective Reynolds number of the simulation. The results are therefore in qualitative agreement with those obtained on the TGV case, the eddy-viscosity models being again too dissipative.

Figure 16(b) shows the effect of the LES models on the resolved scales of the flow. The spectra show that eddy-viscosity models dissipate an important part of the turbulent small scales, reducing their corresponding energy content, and promoting coherent lower scales motions. This effect is particularly visible for the SM model with $C_{S}=0.15$. This can be interpreted as an artificial diminution of the effective Reynolds number of the simulations. On the contrary, the more elaborated models and the RT methodology limits the dissipation at the smallest resolved scales and the locations of the maximum of the spectra are in better agreement with the DNS results. 


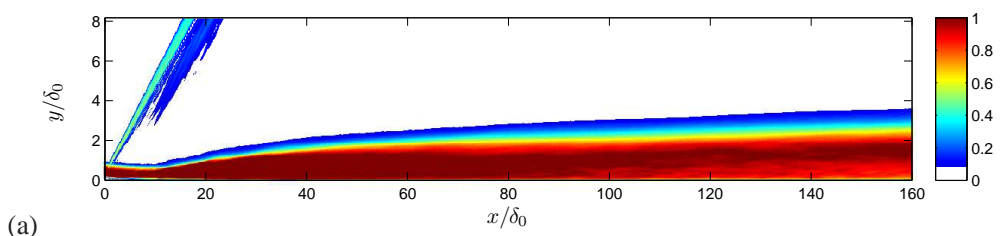

(a)

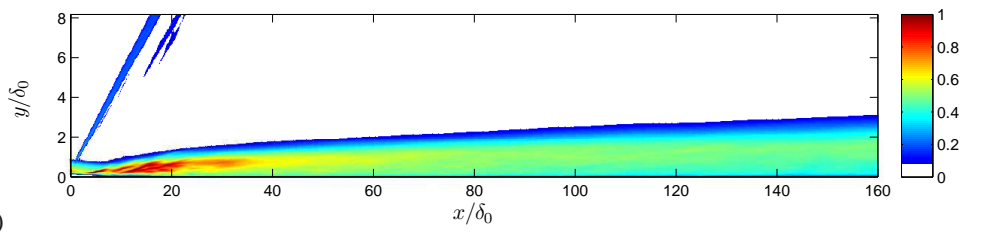

(b)

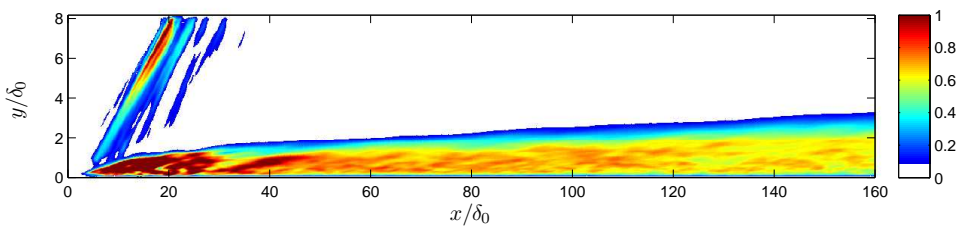

(c)

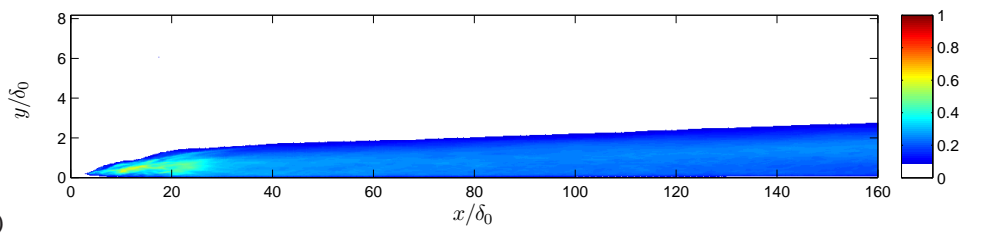

Fig. 15 Influence of the SGS model for the supersonic boundary layer. Colormaps of time-averaged normalized eddy viscosity $v_{t} / v$ (levels between 0 and 1); (a) SM with $C_{S}=0.15$; (b) SM with $C_{S}=0.1$; (c) DSM; (d) MSM-ss. $\delta_{0}$ is the initial boundary layer thickness.

$$
\text { (a) }
$$

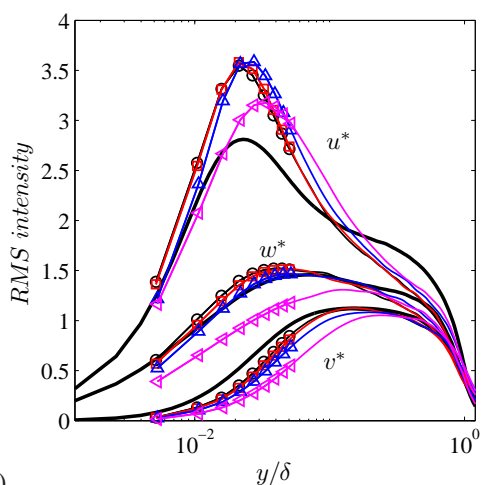

(b)

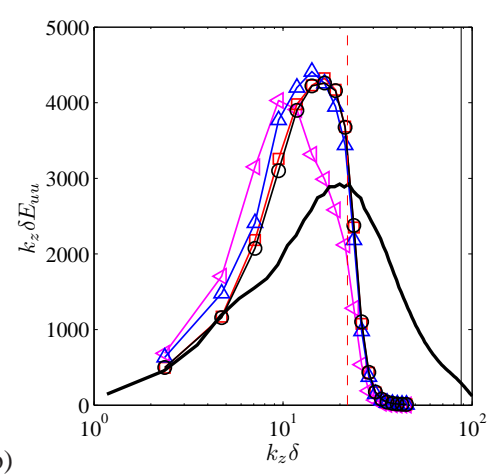

Fig. 16 Influence of the SGS model for the supersonic boundary layer. (a) Density scaled $r m s$ profiles; (b) Pre-multiplied wavenumber spectra for $y / \delta_{99}=0.03$ for the spanwise direction $k_{z}$ (b); ( $)$ DNS; ( $\square$ ) RT methodology; ( $\square$ ) MSM-ss model; ( $\square$ ) DSM model; ( $\square$ ) SM model with $C_{S}=0.15$. The vertical dashed line indicates the approximate cutoff wavenumber for LES3. 


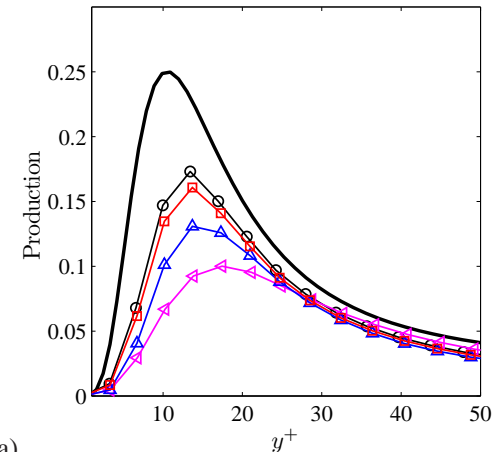

(a)
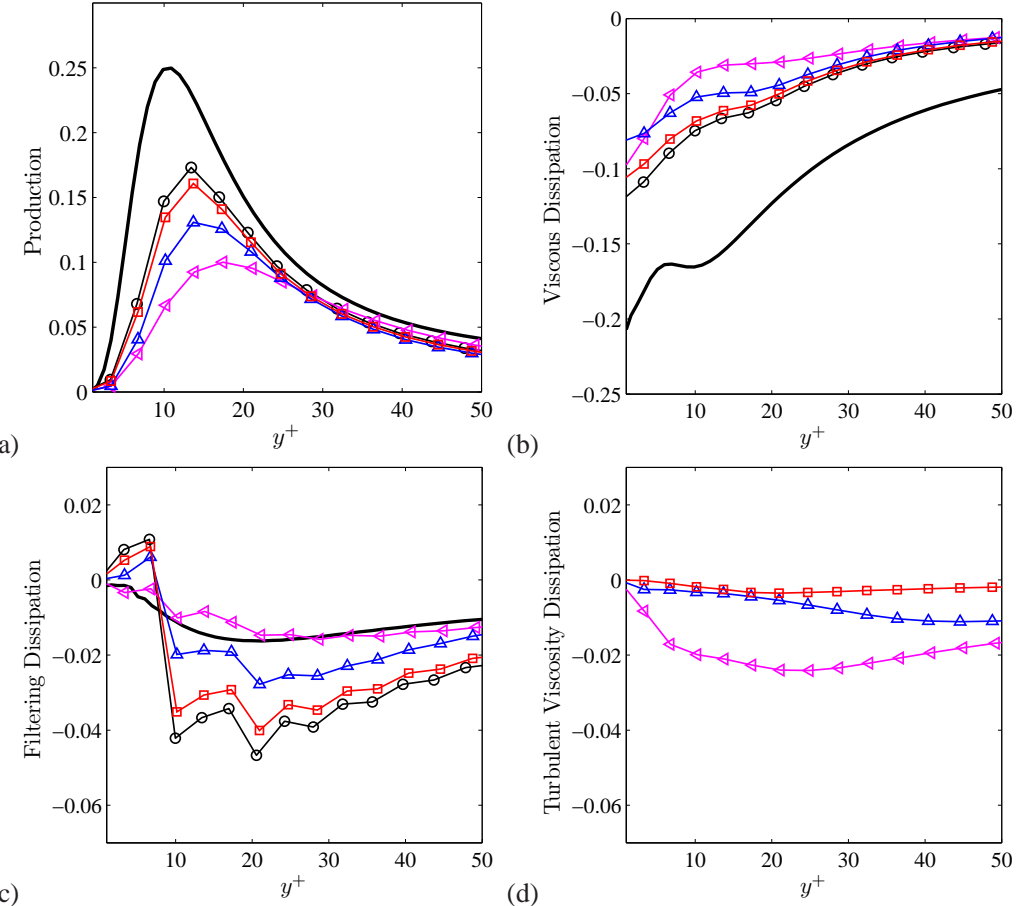

Fig. 17 Influence of the SGS model for the supersonic boundary layer. Turbulent kinetic energy budget: (a) Production term; (b) Viscous dissipation term. (c) Selective filtering dissipation term; (d) SGS dissipation of the LES models; ( $)$ DNS; ( $\square$ ) RT methodology; ( $\square$ ) MSM-ss model; ( $\square$ ) DSM model; ( $\square$ ) SM model with $C_{S}=0.15$.

Figure 17(a) shows that the overdissipation of the eddy-viscosity models reduces the amplitude of the production term, leading to a subsequent diminution of the viscous dissipation term (Fig.17(b)). Again, comparable results are obtained between the RT and MSM-ss methodologies. The dissipation linked to the filtering activity is presented in Fig.17(c). The presence of a SGS model significantly reduces the influence of the selective filter, and this reduction is proportional to the subgrid activity of the model (shown in Fig.17(d)). Another automatic adaptation of the filtering activity is noticed in the near wall region, where the reduction of dissipation is in agreement with wall models. A weak anti-dissipative-like behaviour is even observed for the first points near the wall, as in Stolz et al. [26], maybe due to the particular wall treatment such as the non-centered filters.

Finally, Table 2 gives the computational cost of the different models tested for the supersonic boundary layer. A supplementary cost from around $4 \%$ for a simple Smagorinsky model to almost $30 \%$ for its dynamic version are noted. The cost and the simplicity are thus arguments in favor of the filter-based strategy. 


\begin{tabular}{|c|c|c|c|c|}
\hline Model & RT & SM & MSM & DSM \\
\hline CPU time per iteration & $35.32 \mathrm{~s}$ & $36.63 \mathrm{~s}$ & $39.84 \mathrm{~s}$ & $45.38 \mathrm{~s}$ \\
\hline Base 100 & 100 & 103.7 & 112.8 & 128.5 \\
\hline
\end{tabular}

Table 2 Calculation costs for LES3 simulations (5.88 million points on 72 proc).

\section{Conclusions}

Subgrid-scale (SGS) viscosity models and selective filtering strategy have been compared for quasi-incompressible and compressible large eddy simulations (LES). The first investigated configuration is the Taylor-Green vortex, which constitutes a prototype for transition toward small-scale turbulence. The most common SGS models are implemented and compared to the filter-based strategy, where the filtering alone provides an implicit model to take into account the dissipative effect at small scales. The best results are obtained for the filter-based strategy for a minimal cost. The most sophisticated models, such as the similarity model or the multiscale model in conjunction with a dynamic Smagorinsky model, also give satisfactory results but at the price of an increased effort. In this configuration, the effects of the dispersion error due to the centered finite-difference scheme and of the dissipation error due to the high-order filter are investigated. It is shown that their influence can be greater than the contribution of the SGS models. The analysis of numerical errors is more tricky for complex inhomogeneous flows. The results obtained for a supersonic turbulent boundary layer show that eddy-viscosity models, even in a dynamic form, overdissipate a part of the resolved scales and artificially reduce the effective Reynolds number of the simulations. A multiscale model can provide satisfactory results but fails to improve the implicit methodology based on explicit filtering, which appears as the most efficient.

Similarly, Visbal \& Rizzetta [40], Schlatter et al. [57], Bogey \& Bailly [28] and Kawai et al. [58] also obtained better results without adding an explicit SGS model than with SGS models when using high-order central differences and low-pass filtering schemes. However, as noticed by Kawai et al. [58], the relative roles played by the SGS model, low-pass filtering and the numerical dissipation depend on the numerical scheme, the grid resolution, and the Reynolds number, and further investigations notably for high Reynolds number flows are needed. It would be interesting for instance to qualify the action of the filtering dissipation by a priori analyses as in Fauconnier et al. [45], by designing new filters with some constraints mimicking spectral viscosity or hyperviscosity as in Lamballais et al. [59], or by computing dynamically the filtering amplitude as in Stolz et al. [14]. Further investigations of the interaction with discretization errors are also needed. Since the filter is still active to suppress grid-to-grid oscillations when explicit SGS models are used, it would be interesting to use skew-symmetric form of the derivatives or discrete entropy-preserving form of the energy equation [60] to avoid recourse to the filter as numerical dissipation and, in this way, to separate more clearly its effects as implicit model.

Acknowledgements This work was granted access to the HPC resources of IDRIS and CCRT under the allocation 2011-1736 made by GENCI (Grand Equipement National de Calcul Intensif). The authors acknowledge the support of the French Agence Nationale de la Recherche (ANR) under grant SPICEX (ANR07-CIS7-009). 


\section{References}

1. E. Garnier, N. Adams, and P. Sagaut. Large eddy simulation for compressible flows. Springer, 2009.

2. J. Meyers and P. Sagaut. Is plane channel flow a friendly case for the testing of the large-eddy simulation subgrid-scale models? Phys. Fluids, 19:048105, 2007.

3. S.T. Bose, P. Moin, and D. You. Grid-independent large-eddy simulation using explicit filtering. Phys. Fluids, 22:105103, 2010.

4. S. Radhakrishnan and J. Bellan. Explicit filtering to obtain grid-spacing-independent and discretizationorder-independent large-eddy simulation of compressible single-phase flow. J. Fluid Mech., 697:399435, 2012.

5. G. De Stefano and O.V. Vasilyev. Sharp cutoff versus smooth filtering in large eddy simulation. Phys. Fluids, 14(1):362-369, 2004.

6. X. Yang and S. Fu. The effect of filtering on truncated Navier-Stokes equations. J. Turbulence, 8(8):1-18, 2007.

7. S. Ghosal. An analysis of numerical errors in Large-Eddy Simulations of turbulence. J. Comput. Phys., 125:187-206, 1996.

8. A.G. Kravchenko and P. Moin. On the effect of numerical errors in Large Eddy Simulations of turbulent flows. J. Comput. Phys., 131:310-322, 1997.

9. J.S. Smagorinsky. General circulation experiments with the primitive equations: I. the basic experiment. Mon. Weath. Rev., 91:99-163, 1963.

10. M. Germano, U. Piomelli, P. Moin, and W.H. Cabot. A dynamic subgrid-scale eddy viscosity model. Phys. Fluids A, 3(7):1760-1765, 1991.

11. C. Bogey and C. Bailly. Decrease of the effective Reynolds number with eddy-viscosity subgrid-scale modelling. AIAA Journal, 43(2):437-439, 2005.

12. J. Bardina, J.H. Ferziger, and W.C. Reynold. Improved subgrid scale models for large eddy simulation. AIAA Paper 80-1357, 1980.

13. T.J.R. Hughes, L. Mazzei, and A.A. Oberai. The multiscale formulation of large eddy simulation : Decay of homogeneous isotropic turbulence. Phys. Fluids, 13(2):505-512, 2001.

14. S. Stolz, N.A. Adams, and L. Kleiser. An approximate deconvolution model for large-eddy simulation with application to incompressible wall-bounded flows. Phys. Fluids, 13(4):997-1015, 2001.

15. S. Stolz, N.A. Adams, and L. Kleiser. The approximate deconvolution model for large-eddy simulations of compressible flows and its application to shock-turbulent boundary-layer interaction. Phys. Fluids, 13(10):2985-3001, 2001.

16. J. Gullbrand and F.K. Chow. The effect of numerical errors and turbulence models in large-eddy simulations of channel flow, with and without explicit filtering. J. Fluid Mech., 495:323-341, 2003.

17. V.V. Borue and S.A. Orszag. Self-similar decay of three-dimensional homogeneous turbulence with hyperviscosity. Phys. Rev. E, 51(2):R856-R859, 1995.

18. A.W. Cook and W.H. Cabot. A high-wavenumber viscosity for high-resolution numerical methods. $J$. Comput. Phys., 195(2):594-601, 2004.

19. A.G. Lamorgese, D.A. Caughtey, and S.B. Pope. Direct numerical simulation of homogeneous turbulence with hyperviscosity. Phys. Fluids, 17:015106, 2005.

20. G.-S. Karamanos and G.E. Karniadakis. A spectral-vanishing viscosity method for large-eddy simulations. J. Comput. Phys., 163:22-50, 2000.

21. J.P. Boris, F.F. Grinstein, E.S. Oran, and R.L. Kolbe. New insights into large eddy simulation. Fluid Dyn. Res., 10:199-228, 1992.

22. C. Fureby and F.F. Grinstein. Large eddy simulation of high-Reynolds number free and wall-bounded flows. J. Comput. Phys., 181:68-97, 2002.

23. J.A. Domaradzki and E.M. Saiki. A subgrid-scale model based on the estimation of unresolved scales of turbulence. Phys. Fluids, 9(7):2148-2164, 1997.

24. J.A. Domaradzki and K.-C. Loh. The subgrid-scale estimation model in the physical-space representation. Phys. Fluids, 11(8):2330-2342, 1999.

25. J.A. Domaradzki and P.P. Yee. The subgrid-scale estimation model for high Reynolds number turbulence. Phys. Fluids, 12(1):193-196, 2000.

26. S. Stolz and N.A. Adams. Large-eddy simulation of high-Reynolds-number supersonic boundary layers using the approximate deconvolution model and rescaling and recycling technique. Phys. Fluids, 15(8):2398-2412, 2003.

27. D.P. Rizzetta, M.R. Visbal, and G.A. Blaisdell. A time-implicit high-order compact differencing and filtering scheme for large-eddy simulation. Int. J. Numer. Meth. Fluids, 42:665-693, 2003.

28. C. Bogey and C. Bailly. Large eddy simulations of round jets using explicit filtering with/without dynamic Smagorinsky model. International Journal of Heat and Fluid Flow, 27:603-610, 2006. 
29. C. Bogey and C. Bailly. Large eddy simulations of transitional round jets: Influence of the Reynolds number on flow development and energy dissipation. Phys. Fluids, 18:065101, 2006.

30. J. Mathew, R. Lechner, H. Foysi, J. Sesterhenn, and R. Friedrich. An explicit filtering method for large eddy simulation of compressible flows. Phys. Fluids, 15(8):2279-2289, 2003.

31. M.R. Visbal, P.E. Morgan, and D.P. Rizzetta. An implicit LES approach based on high-order compact differencing and filtering schemes. AIAA Paper 2003-4098, 2003.

32. J. Berland, P. Lafon, F. Daude, F. Crouzet, C. Bogey, and C. Bailly. Filter shape dependence and effective scale separation in large-eddy simulations based on relaxation filtering. Computers and Fluids, 47:6574, 2011.

33. B. Vreman, B. Geurts, and H. Kuerten. Large-eddy simulation of the turbulent mixing layer. J. Fluid Mech., 339:357-390, 1997.

34. B. Vreman, B. Geurts, and H. Kuerten. Subgrid-modelling in LES of compressible flow. Appl. Sci. Res., 54:181-203, 1995.

35. C. Bogey and C. Bailly. A family of low dispersive and low dissipative explicit schemes for noise computation. J. Comput. Phys., 194:194-214, 2004.

36. C. Bogey, N. De Cacqueray, and C. Bailly. A shock-capturing methodology based on adaptative spatial filtering for high-order non-linear computations. J. Comput. Phys., 228(5):1447-1465, 2009.

37. A. Yoshizawa. Statistical theory for compressible turbulent shear flows, with the application to subgrid modeling. Phys. Fluids, 29:2152-2164, 1986.

38. G. Erlebacher, M.Y. Hussaini, C.G. Speziale, and T.A. Zang. Toward the large-eddy simulation of compressible turbulent flows. J. Fluid Mech., 238:155-185, 1992.

39. C. Meneveau and J. Katz. Scale-invariance and turbulence models for large-eddy simulation. Ann. Rev. Fluid Mech., 32:1-32, 2000.

40. M.R. Visbal and D.P. Rizzetta. Large-Eddy Simulation on curvilinear grids using compact differencing and filtering schemes. ASME Journal of Fluids Engineering, 124:836-847, 2002.

41. S. Hickel, N.A. Adams, and J.A. Domaradzki. An adaptive local deconvolution method for implicit LES. J. Comput. Phys., 213(1):413-436, 2006.

42. D. Drikakis, C. Fureby, F.F. Grinstein, and D. Youngs. Simulation of transition and turbulence decay in the Taylor-Green vortex. J. Turbulence, 8(20):1-12, 2007.

43. A.J. Chandy and S.H. Frankel. Regularization-based sub-grid scale (SGS) models for large eddy simulations (LES) of high-Re decaying isotropic turbulence. J. Turbulence, 10(25):1-22, 2009.

44. D. Fauconnier, C. De Langhe, and E. Dick. Construction of explicit and implicit dynamic finite difference schemes and application to the large-eddy simulation of the Taylor-Green vortex. J. Comput. Phys., 228:8053-8084, 2009

45. D. Fauconnier, C. Bogey, and E. Dick. On the performance of relaxation filtering for large-eddy simulation. J. Turbulence, 14(1):22-49, 2013.

46. M. E. Brachet, D. I. Meiron, S. A. Orszag, B. G. Nickel, R. H. Morf, and U. Frisch. Small-scale structure of the Taylor-Green vortex. J. Fluid Mech., 130:411-452, 1983.

47. C.K.W. Tam and J.C. Webb. Dispersion-relation-preserving finite difference schemes for computational acoustics. J. Comput. Phys., 107:262-281, 1993.

48. J. Berland, C. Bogey, O. Marsden, and C. Bailly. High-order, low dispersive and low dissipative explicit schemes for multiple-scale and boundary problems. J. Comput. Phys., 224(2):637-662, 2007.

49. K.W. Thompson. Time dependent boundary conditions for hyperbolic systems. J. Comput. Phys., 68:124, 1987.

50. X. Gloerfelt and P. Lafon. Direct computation of the noise induced by a turbulent flow through a diaphragm in a duct at low Mach number. Computers and Fluids, 37:388-401, 2008.

51. E. J. Hopkins and M. Inouye. An evaluation of theories for predicting turbulent skin friction and heat transfer on flat plates at supersonic and hypersonic Mach numbers. AIAA Journal, 9(3):993-1003, 1971.

52. S. Pirozzoli and M. Bernardini. Direct numerical simulation database for impinging shock wave/turbulent boundary-layer interaction. AIAA Journal, 49(6):1307-1312, 2011.

53. M. Bernardini and S. Pirozzoli. Wall pressure fluctuations beneath supersonic turbulent boundary layers. Phys. Fluids, 23:085102, 2011.

54. P. Schlatter and R. Örlü. Assessment of direct numerical simulation data of turbulent boundary layers. J. Fluid Mech., 659:116-126, 2010.

55. C. Bogey and C. Bailly. Turbulence and energy budget in a self-preserving round jet: direct evaluation using large-eddy simulation. J. Fluid Mech., 627:129-160, 2009.

56. N. A. Adams and S. Stolz. A subgrid-scale deconvolution approach for shock capturing. J. Comput. Phys., 178:391-426, 2002.

57. P. Schlatter, S. Stolz, and L. Kleiser. LES of transitional flows using the approximate deconvolution model. International Journal of Heat and Fluid Flow, 25:549-558, 2004. 
58. S. Kawai, S.K. Shankar, and S.K. Lele. Assessment of localized artificial diffusivity scheme for largeeddy simulation of compressible turbulent flows. J. Comput. Phys., 229(5):1739-1762, 2010.

59. E. Lamballais, V. Fortuné, and S. Laizet. Straightforward high-order numerical dissipation via the viscous term for direct and large eddy simulation. J. Comput. Phys., 230:3270-3275, 2011.

60. A.E. Honein and P. Moin. Higher entropy conservation and numerical stability of compressible turbulence simulations. J. Comput. Phys., 201:531-545, 2004. 